\title{
COMPOSIÇÃO FLORÍSTICA DO PARQUE ESTADUAL MATAS DO SEGREDO, LOCALIZADO NA ÁREA URBANA DO MUNICÍPIO DE CAMPO GRANDE, MATO GROSSO DO SUL.
}

\author{
Ademir Kleber Morbeck de Oliveira ${ }^{1}$; Ubirazilda Maria Rezende ${ }^{2}$
}

\section{RESUMO}

Áreas verdes são elementos que atuam como indicadores da qualidade de vida, estando intimamente ligadas ao lazer da população, além de desempenhar funções estéticas e ecológicas no ambiente urbano. Desta maneira, o conhecimento das espécies existentes nestas áreas é importante para o gerenciamento destes locais. Este trabalho teve como objetivo realizar o levantamento de espécies da flora no Parque Estadual Matas do Segrego (Campo Grande, MS), visando fornecer subsídios a projetos de manejo e recuperação da área. Através de caminhadas aleatórias na área, foram identificadas 188 espécies (63 famílias), sendo Fabacea a com maior riqueza (22 espécies), seguida por Rubiaceae (10), Apocynaceae, Bignoniaceae e Sapindaceae (8), Annonaceae e Euphorbiaceae (7), com 10 espécies pertencentes ao estrato herbáceo, 38, arbustivo, 111, arbóreo, 24, trepadeiras, além de uma espécie de parasita. O ambiente com maior diversidade é o cerrado, com 94 espécies, seguido pelo Cerradão, 56, Campo Sujo e Borda do Cerrado, cada um com 29 espécies, Mata Seca Semidecícua, 22, Mata de Galeria, 20 e, Mata de Galeria Inundável, 11. Levando-se em consideração que 59\% das espécies encontradas são árvores, pode-se considerar o local como sendo, predominantemente, coberto por vegetação de porte arbóreo e apresentando boa diversidade florística.

Palavras-chave: Unidades de Conservação; Parques urbanos; Preservação de Fragmentos.

\section{FLORISTIC COMPOSITION OF MATAS DO SEGREDO STATE PARK, LOCATED IN THE URBAN AREA OF CAMPO GRANDE, MATO GROSSO DO SUL.}

\section{ABSTRACT}

Urban green areas are sites that act as life quality indicator, since they are closely linked to leisure of the population. In this way, they also have aesthetic and ecological functions in the urban environment. Thus, the knowledge about this species is important for the management of these sites. The goal of this study was to identify the flora of Parque Estadual Matas do Segredo (Campo Grande, MS) to provide information to enable management projects focused on recovery. Through random walks in the area, 188 species have been identified (63 families) and Fabaceae had the highest number of species (63 families), followed by Rubiaceae (10), Apocynaceae, Bignoniaceae and Sapindaceae (8), Annonaceae and Euphorbiaceae (7), with 10 herbaceous, 38 shrub, 111 tree, 24 vines and a kind of parasite. The environment with greater diversity of species is the Savanna, with 94, followed by Woody Savanna, 56, Dirty Field and Edge Savanna, each with 29 species, Dry Forest semideciduous, 22, Gallery Forest, 20, and Gallery Forest Wetland , 11. Taking into account that 59\% of the species found are trees, can consider the site as being predominantly covered by arboreal vegetation and presenting good floral diversity.

Key words: Units of Conservation; Urban Parks; Fragments preservations.

\footnotetext{
${ }^{1}$ Professor Dr., Universidade Anhanguera-Uniderp, Programa de Pós-Graduação em Meio Ambiente e Desenvolvimento Regional, Campo Grande-MS. Email: akmorbeck@hotmail.com

2 Professora MSc., Universidade Anhanguera-Uniderp, Curso de Ciências Biológicas, Campo Grande-MS. Email: resende@nin.ufms.br
} 


\section{INTRODUÇÃO}

A presença de vegetação nas áreas urbanas propicia uma série de funções ambientais, como por exemplo, microclima mais ameno, temperatura e umidade relativa mais agradável, diminuindo também o nível de poluição, através da redução do número de partículas sólidas e gasosas do ar, além de abafar os ruídos, com diminuição da poluição sonora; a presença de vegetação também facilita o processo de infiltração no solo das águas pluviais, evitando o escorrimento superficial, além de servir de refúgio à fauna silvestre (TROPPMAIR e GALINA, 2003; SÃO PAULO, 2004).

Estes processos ambientais são facilitados pela presença de áreas verdes, tais como os parques urbanos, que também são locais voltados para atividades de lazer, esportivas, culturais, conservação, entre outras (MACEDO e SAKATA, 2003; MANTOVANI, 2006). Macedo e Sakata (2003) também explicam que estas áreas são "Todo espaço de uso público destinado à recreação de massa, qualquer que seja o seu tipo, capaz de incorporar intenções de conservação e cuja estrutura morfológica e auto-suficiente, isto é, não é diretamente influenciada em sua configuração por nenhuma estrutura construída em seu entorno”.

Toledo e Santos (2012) escrevem que existem uma gama de fatores benéficos que estes locais propiciam, relacionadas ao ambiente urbano, pois as cidades se tornaram cada vez menos atrativas em termos de qualidade ambiental e de vida devido ao aumento de problemas ambientais, tais como a poluição atmosférica, chuva ácida, enchentes, entre outros e amenizar as tensões do cotidiano, proporcionando liberdade e saúde, são alguns propósitos dos parques. A criação destas áreas também tem sido uma estratégia para preservar os remanescentes vegetais no perímetro urbano, permitindo a manutenção da biodiversidade, mesmo em regiões densamente povoadas.

Porém poucas são as cidades que possuem remanescentes vegetais relevantes dentro da área urbana. Campo Grande, capital do Estado de Mato Grosso do Sul, é considerada uma das mais arborizadas do país, uma exceção nesse sentido, possuindo uma grande diversidade de áreas verdes (CAMPO GRANDE, 1999). Pela sua posição geográfica, está localizada em área de Cerrado, que inclui um grupo de formas de vegetação com diferentes fisionomias marcadas pela presença de uma camada herbácea parcial ou contínua e por uma proporção variada de espécies lenhosas, englobando campo limpo, campo sujo, campo cerrado, cerrado e cerradão. Estes gradientes fisionômicos têm sido interpretados como resultantes do nível de nutrientes no solo, que aumenta das áreas abertas em direção ao cerradão, sendo acompanhadas por mudanças na composição de espécies, resultando em um dos biomas mais ricos do Brasil (SANO e ALMEIDA, 1998; SCARIOT et al., 2005).

Uma das áras verdes mais importantes da capital é o atual Parque Estadual Matas do Segredo, conhecido na década de 1980 como Chácara Santa Inês, localizado ao norte da cidade. Esta região confunde-se com a história da colônia japonesa, que migrou para Campo Grande utilizando a antiga Estrada de Ferro Noroeste do Brasil e se instalou no local, através da compra de propriedades para a produção de hortifrutigranjeiros. Parte das propriedades foi posteriormente vendida e remembradas com o nome de Chácara Santa Inês, que depois foi adquirida pelo extinto Instituto de Previdência Social de Mato Grosso (Previsul), que pretendia utilizá-lo para a construção de conjuntos residenciais. Porém tal ação não foi concretizada graças à intervenção de associações de moradores de bairros próximos, ambientalistas, Associação de Hortifrutigranjeiros de Campo Grande e Centro de Abastecimento de Mato Grosso do Sul, além da organização não governamental Ecologia e Ação (Ecoa), que criaram um movimento para impedir o desmatamento da área, além de propor a desapropriação do local e a criação de um Jardim Botânico para a capital. Porém devido a questões legais relacionadas à posse da área, entre outros problemas burocráticos, o local ficou abandonado por alguns anos, sofrendo pressões por parte da população do entorno, já bastante urbanizado com

Ademir Kleber Morbeck de Oliveira e Ubirazilda Maria Rezende 
grandes bairros, como Campo Belo e Jardim Presidente, o que resultou em caça e extração de produtos florestais, além de diversos incêndios no local, resultado da ação antrópica. Como o combate aos incêndios era complexo devido à dificuldade de acesso a diversos pontos, foi realizada a abertura de trilhas para dar passagem aos veículos do corpo de bombeiros. A descaracterização do local foi também acelerada por esta ação, que permitiu maior facilidade de acesso a caçadores e coletores da região.

Após vários anos de abandono e depredação, em 18 de novembro de 1990 foi assinada uma proposta para transformar o local em Unidade de Conservação, levando-se em conta que a região apresenta grande importância como manancial de água, formador do córrego Prosa, um dos mais importantes do município. De acordo com relatos de antigos moradores, existiam 33 pequenas nascentes, que foram desaparecendo ao longo do tempo devido ao processo de desmatamento do entorno das nascentes e posterior assoreamento dos locais, principalmente devido ao solo arenoso, predominante na região. Este fator estava contribuindo para o desaparecimento da história da cidade, fundada nas margens do córrego Prosa, desde sua confluência com o córrego Segredo (CAMPO GRANDE, 1999), cujas nascentes estão dentro do Parque Estadual Matas do Segredo.

O processo de criação da área de preservação foi somente efetivado quando a Secretaria de Estado de Meio Ambiente (Sema) celebrou um instrumento de comodato com o Previsul, e o governador criou o Jardim Botânico de Campo Grande pelo decreto estadual n ${ }^{\circ}$ 7. 119/93 de
17 de março de 1993, com as seguintes justificativas: a “obrigatoriedade da preservação da biodiversidade do cerrado”; “a importância da preservação da nascente do córrego Segredo, pelo seu valor histórico, ecológico e pela necessidade da manutenção da qualidade de suas águas”; “a carência por parte da população campograndense de áreas naturais primitivas, que atendam aos objetivos educacionais e de lazer”; “as manifestações em prol da conservação da área e a política preservacionista adotada pelo Governo Estadual”; e o comodato celebrado entre a Sema e o Previsul. $\mathrm{O}$ artigo $2^{\circ}$ desse mesmo decreto atribuiu a Sema a competência para administrar e, no prazo máximo de 180 dias, para instituir o regimento de funcionamento da área, o que não ocoreu.

Em 2000, o governo estadual transformou o Jardim Botânico de Campo Grande em Parque Estadual Matas do Segredo (decreto $\mathrm{n}^{\circ} 9.935$ de 05 de junho de 2000), com área de 177,58 ha e “o objetivo de preservar amostras de cerrado e matas nela associadas, espécies da flora e fauna, a manutenção de bacias hidrográficas, e valorização do patrimônio paisagístico e cultural do município de Campo Grande, objetivando sua utilização para fins de pesquisa científica, educação ambiental, recreação e turismo em contato com a natureza” (Diário Oficial n ${ }^{\circ}$ 5.279, p. 5, de 6 de junho de 2000).

Levando-se em consideração que a caracterização da vegetação existente nas áreas verdes inseridas na malha urbana é necessária e urgente para um melhor conhecimento das espécies vegetais constituintes desses remanescentes, o objetivo deste trabalho foi realizar o levantamento de espécies da flora existentes no Parque Estadual Matas do Segredo.

\section{MATERIAL E MÉTODOS}

A área de estudo é o Parque Estadual Matas do Segredo com 177,58 ha, localizado na área urbana do município de Campo Grande-MS (Figura 1), cerca de 20²6’34” de latitude e $54^{\circ} 38^{\prime} 47^{\prime \prime}$ de longitude, fazendo limites com bairros residenciais ao sul, sudeste, leste e nordeste e, áreas de pastagens ao norte, noroeste, oeste e sudoeste. $\mathrm{O}$ clima da região é do tipo Aw, de acordo com a classificação de Köppen (1948), com invernos secos e verões chuvosos. A precipitação pluvial média é em torno de $1500 \mathrm{~mm}$, com os meses de menor precipitação de junho a agosto (MATO GROSSO DO SUL, 1990). 
Figura 1. Parque Estadual Matas do Segredo, Campo Grande, Mato Grosso do Sul, com diferentes formas de vegetação Figura 1. Matas do Segredo State Park, Campo Grande, Mato Grosso do Sul, with different vegetation types

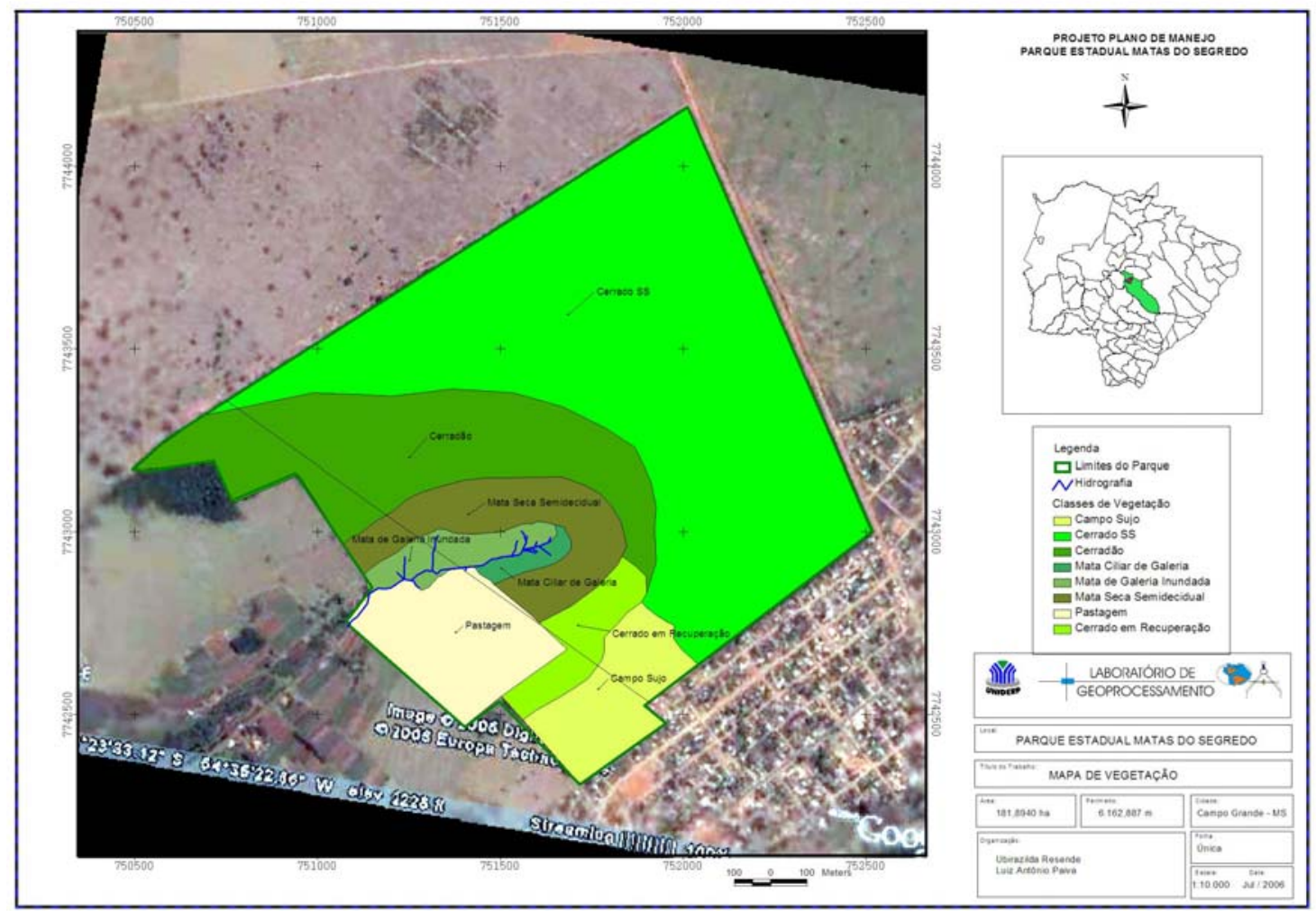

Fonte: Projeto Plano de Manejo - Universidade Anhanguera-Uniderp, Laboratório de Geoprocessamento.

A área está representada por formações de cerrado, cerradão e mata de galeria do córrego Segredo, sendo consideradas como árvores os indivíduos lenhosos sem ramificações na base e altura superior a $2 \mathrm{~m}$; como arbustos, indivíduos lenhosos, com ou sem ramificações na base, e altura entre 0,50 a $2 \mathrm{~m}$ e, estrato herbáceo, indivíduos de altura até $50 \mathrm{~cm}$ do solo (HERINGER et al., 1977).

Durante caminhadas aleatórias na área, realizadas mensalmente durante os anos de 2008 e 2009, totalizando 12 visitas ao local, abrangendo todas as formações encontradas, foram coletados prioritariamente materiais férteis de todas as angiospermas, incluindo o hábito arbóreo-arbustivo e subarbustivo-herbáceo, para posterior herborização. Eventualmente foram coletados espécimes estéreis, para informações complementares.

Os espécimes coletados foram previamente desidratados, conforme procedimento convencional e depositadas na Coleção Botânica da Universidade Anhangura-Uniderp e no Herbário da Universidade Federal de Mato Grosso do Sul - UFMS, sendo os materiais identificados através de chaves de identificação, bibliografias especializadas, comparação com materiais de herbários e consultas a especialistas, com a nomenclatura utilizada para classificar as espécies seguindo o padrão do Missouri Botanical Garden e APG-II (2003). 
A florística realizada no local denota a presença de seis tipos fitofisionômicos descritos para o bioma Cerrado (SANO e ALMEIDA, 1998; SCARIOT et al., 2005), com aproximadamente $80 \%$ da área do parque representada pelas formações não associadas aos cursos d'água, ou seja, o Cerrado, que varia entre stricto senso e formas de lato sensu (Campo Sujo, Cerradão e Mata Seca Semidecidual) e os $20 \%$ restantes, contemplando áreas de Mata de Galeria e Mata de Galeria Inundável.

A formação de cerrado stricto senso apresenta uma área de grande influência antrópica, pois constitui basicamente o limite entre o entorno antrópizado e a área de mata onde se localizam as nascentes do córrego Segredo.

A ação antrópica sofrida ao longo dos anos descaracterizou algumas áreas do local, transformando-as em Campo Sujo, onde pode-se observar uma concentração de espécies oportunistas e invasoras com formação de clareiras, predominando o estrato graminoso (Brachiaria spp.) e alguns representantes das Cyperaceae, além de espécies oportunistas.

O Cerradão e a Mata Seca Semidecidual destacam-se pela riqueza de espécies e por constituir uma importante faixa de proteção das nascentes do Córrego Segredo, servindo ainda como refúgio da fauna ali existente. Dentre as formações vegetais do Cerrado, a Mata de Galeria e a Mata de Galeria Inundável são de extrema importância por estarem associadas aos cursos d'água. Estas formações destacam-se pela riqueza e diversidade genética e pelo seu papel na proteção dos recursos hídricos, edáficos, fauna silvestre e aquática. A composição das espécies na área de Mata de Galeria, bem como, na Mata de Galeria Inundável, é distinta da composição das vegetações circunvizinhas, resultado muito provável da influência dos fatores antrópicos, edáficos e hidrogeomorfológicos (SANO e ALMEIDA, 1998; SCARIOT et al., 2005).

A presença de antigos sítios e chácaras na região explica a ocorrência, em alguns locais de mata, de exemplares de espécies exóticas, tais como Mangifera indica L. (Família Anacardiaceae), uma espécie arbórea encontrada na Mata Seca Semidecídua, Asclepia curasavica Mart. (Família
Asclepiadaceae), arbustos encontrados no Campo Sujo, Senecio sp. (Família Asteraceae), arbustos presentes no Campo Sujo e Mata de Galeria Inundável, Adenanthera pavonina L. (Família Fabaceae), árvores encontradas no Campo Sujo, Brachiaria humidicola (Rendle) Schweick. e B. decumbens Stapf (Família Poaceae), gramíneas encontradas na Borda do Cerrado, Campo Sujo, Cerrado e Cerradão e, Eucalyptus sp. (Família Myrtaceae), árvores localizadas na Borda do Cerrado.

Estas espécies, indicadoras da ocupação humana na região, apresentam efeitos negativos sobre a diversidade de espécies nativas, principalmente as braquiárias sobre o estrato herbáceo, devido a capacidade competitiva desta gramínea, o que leva ao desaparecimento das espécies autóctones. Apesar deste processo de ocupação, o tempo que o local permaneceu fechado para atividades econômicas permitiu que a mata original ocupasse parcialmente os locais que haviam sido anteriormente modificados, propiciando uma formação secundária que se encontra em diferentes estágios de regeneração.

Porém em muitos locais, a ação antrópica ocorrida, com a retirada seletiva de madeira para construção civil, lenha para carvoarias, ação de raízeiros, queimadas, além de outros impactos, levou a uma predominância de espécies consideras pioneiras de diferentes formações vegetacionais, de crescimento a pleno sol (heliofilas), como por exemplo, Allophyllus edulis (Mata Seca), Anadenanthera falcata (Cerrado), Annona coriacea (Cerrado), Aspidosperma macrocarpon (Cerrado), Astronium fraxinifolium (Cerrado), Aloysia virgata (Mata Seca), Cordia glabrata (Mata Seca), Cecropia pachystachya (Mata de Galeria, Mata Seca, Cerrado), Couepia grandiflora (Mata de Galeria, Cerrado), Curatella americana (Cerrado), Croton urucurana (Mata de Galeria), Casearia sylvestris (diferentes formações), Dalbergia miscolobium (Cerrado), Dendropanax cuneatum (Mata de Galeria), Dipteryx alata (Cerrado), Dilodendron bipinnatum (Mata de Galeria) Diptychandra aurantiaca (Mata Seca, Cerradão), Eriotheca gracilipes (Cerrado, Mata de Galeria), Guarea guidonia (Mata de Galeria), Gochnatia polymorpha (Cerrado), Hancornia 
speciosa (Cerrado), Handroanthus impetiginosus (Cerradão, Mata Seca), H. aureus (Cerrado), Kielmeyera variabilis (Cerrado), Lafoensia pacari (Cerrado), Luehea divaricata (Mata de Galeria), Myracrodruon urundeuva (Mata Seca, Cerradão), Plathymenia reticulata (Mata Seca, Cerrado), Pouteria torta (Cerrado), Qualea parviflora (Cerrado), Rapanea ferruginea (Mata de Galeria), Schinus terebinthifolius (Cerradão, Mata Seca), Stryphnodendron adstringens (Cerrado), Sapindus saponaria (Mata de Galeria), Tapirira guianensis (Mata de Galeria, Cerrado), Trema micrantha (Mata Seca), Vochysia tucanorum (Mata de Galeria, Cerrado), Xylopia emarginata (Mata de Galeria), entre outras (LORENZI, 2008ab, 2009).

No total, foram identificadas 188 espécies no Parque, pertencentes a 63 famílias, sendo 10 do estrato herbáceo,
38, arbustivo, 111, arbóreo, 24, trepadeiras, além de uma espécie de parasita. O ambiente com maior diversidade de espécies é o Cerrado, com 94, seguido pelo Cerradão, 56, Campo Sujo e Borda do Cerrado, cada um com 29 espécies, Mata Seca Semidecícua, 22, Mata de Galeria, 20 e, Mata de Galeria Inundável, 11 (Tabela 1).

A área de Cerrado stricto sensu é a mais representativa, em termo de espécies, com 50\%, seguido pela área de Cerradão, com 29,8\%. Levando-se em consideração que $59 \%$ das espécies encontradas são árvores, pode-se considerar o local como sendo, predominantemente, coberto por vegetação de porte arbóreo, uma indicação que o processo de sucessão se apresenta em estágio adiantado, eliminado espécies herbáceas e arbustivas (25\%), que seriam mais comuns em áreas em estágio inicial de sucessão.

Tabela 1. Listagem de espécies amostradas no Parque Estadual Matas do Segredo, Campo Grande, MS, com sua distribuição por porte e ocorrência

Table 1. List of species sampled at Parque Estadual Matas do Segredo, Campo Grande, MS, with distribuition of species by size and occurrence

\begin{tabular}{|c|c|c|c|c|c|c|c|c|c|c|c|c|c|}
\hline \multirow[b]{2}{*}{ Família } & \multirow[b]{2}{*}{ Espécie } & \multicolumn{5}{|c|}{ PORTE } & \multicolumn{7}{|c|}{ OCORRÊNCIA } \\
\hline & & $\sum_{\text {| }}^{\pi}$ & 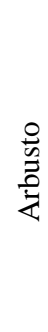 & $\begin{array}{l}\stackrel{0}{0} \\
\text { 总 } \\
\text { 它 }\end{array}$ & 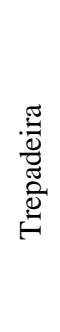 & 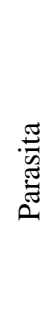 & 율 & Ů & 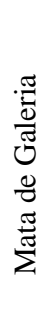 & 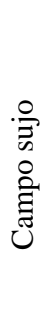 & 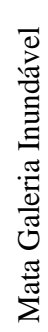 & 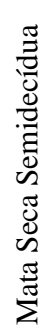 & 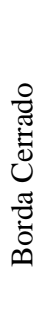 \\
\hline \multirow[t]{4}{*}{ Anacardiaceae } & $\begin{array}{c}\text { Astronium } \\
\text { fraxinifolium } \\
\text { Schott ex Spreng. }\end{array}$ & & & $\mathrm{X}$ & & & & $\mathrm{X}$ & & & & & \\
\hline & $\begin{array}{c}\text { Myracrodruon } \\
\text { urundeuva } \\
\text { Allemão }\end{array}$ & & & $\mathrm{X}$ & & & & $\mathrm{X}$ & & & & & \\
\hline & $\begin{array}{c}\text { Schinus } \\
\text { terebinthifolius } \\
\text { Raddi }\end{array}$ & & & $\mathrm{X}$ & & & $\mathrm{X}$ & & $\mathrm{X}$ & & & & \\
\hline & $\begin{array}{c}\text { Tapirira } \\
\text { guianensis Aubl. }\end{array}$ & & & $\mathrm{X}$ & & & $\mathrm{X}$ & & $\mathrm{X}$ & & & & \\
\hline Annonaceae & Annona & & & $\mathrm{X}$ & & & $\mathrm{X}$ & $\mathrm{X}$ & & & & & \\
\hline
\end{tabular}




\begin{tabular}{|c|c|c|c|c|c|c|c|c|c|c|c|}
\hline & crassiflora Mart. & & & & & & & & & & \\
\hline & $\begin{array}{c}\text { Annona coriacea } \\
\text { Mart. }\end{array}$ & & & $\mathrm{X}$ & & $\mathrm{x}$ & $\mathrm{X}$ & & & & \\
\hline & $\begin{array}{c}\text { Duguetia } \\
\text { furfuracea (A. St.- } \\
\text { Hil.) Saff. }\end{array}$ & & $\mathrm{X}$ & & & & & & $\mathrm{X}$ & & $\mathrm{x}$ \\
\hline & $\begin{array}{c}\text { Rollinia } \\
\text { emarginata } \\
\text { Schltdl }\end{array}$ & & & $\mathrm{x}$ & & & $\mathrm{X}$ & $\mathrm{X}$ & & & \\
\hline & $\begin{array}{c}\text { Unonopsis } \\
\text { lindmanii R.E. Fr. }\end{array}$ & & & $\mathrm{x}$ & & & $\mathrm{X}$ & $\mathrm{X}$ & & & \\
\hline & $\begin{array}{l}\text { Xylopia aromatica } \\
\text { (Lam.) Mart. }\end{array}$ & & & $\mathrm{x}$ & & $\mathrm{x}$ & $\mathrm{X}$ & & & & \\
\hline & $\begin{array}{c}\text { Xylopia } \\
\text { emarginata Mart. }\end{array}$ & & & $\mathrm{x}$ & & & & & & $\mathrm{x}$ & \\
\hline Alismataceae & $\begin{array}{c}\text { Echinodorus } \\
\text { grandiflorus } \\
\text { (Cham.\& Schltdl.) } \\
\text { M. }\end{array}$ & $\mathrm{X}$ & & & & & & & & $\mathrm{X}$ & \\
\hline \multirow[t]{8}{*}{ Apocynaceae } & $\begin{array}{c}\text { Aspidosperma } \\
\text { macrocarpon } \\
\text { Mart. }\end{array}$ & & & $\mathrm{x}$ & & $\mathrm{x}$ & $\mathrm{X}$ & & & & \\
\hline & $\begin{array}{c}\text { Aspidosperma } \\
\text { tomentosum Mart. }\end{array}$ & & & $\mathrm{X}$ & & $\mathrm{x}$ & & & $\mathrm{X}$ & & \\
\hline & $\begin{array}{c}\text { Hancornia } \\
\text { speciosa Gomes }\end{array}$ & & & $\mathrm{x}$ & & $\mathrm{x}$ & $\mathrm{X}$ & & & & \\
\hline & Metastelma sp. & & & & $\mathrm{X}$ & & & & & & $\mathrm{X}$ \\
\hline & Orthosia sp. & & & & $\mathrm{X}$ & & & & & & $\mathrm{X}$ \\
\hline & $\begin{array}{l}\text { Prestonia calycina } \\
\text { Müll. Arg. }\end{array}$ & & & & $\mathrm{X}$ & & & & $\mathrm{X}$ & & $\mathrm{X}$ \\
\hline & $\begin{array}{c}\text { Rhodocalyx } \\
\text { rotundifolius Müll. } \\
\text { Arg. }\end{array}$ & & $\mathrm{X}$ & & & $\mathrm{x}$ & & & $\mathrm{X}$ & & \\
\hline & $\begin{array}{c}\text { Secondatia } \\
\text { densiflora A. DC. }\end{array}$ & & & & $\mathrm{X}$ & & & & $\mathrm{X}$ & & $\mathrm{X}$ \\
\hline \multirow[t]{2}{*}{ Araliaceae } & $\begin{array}{c}\text { Didymopanax } \\
\text { macrocarpus } \\
\text { (Cham. \& Schltdl.) } \\
\text { Seem }\end{array}$ & & & $\mathrm{x}$ & & $\mathrm{x}$ & & & $\mathrm{X}$ & & \\
\hline & $\begin{array}{c}\text { Dendropanax } \\
\text { cuneatus (DC.) } \\
\text { Decne. \& Planch. }\end{array}$ & & & $\mathrm{X}$ & & & & & & $\mathrm{X}$ & \\
\hline
\end{tabular}




\begin{tabular}{|c|c|c|c|c|c|c|c|c|c|c|c|c|}
\hline \multirow[t]{4}{*}{ Asteraceae } & $\begin{array}{l}\text { Achyrocline } \\
\text { satureioides } \\
\text { (Lam.) DC. }\end{array}$ & & $\mathrm{X}$ & & & & & & $\mathrm{X}$ & & & $\mathrm{X}$ \\
\hline & Aspilia sp. & $\mathrm{X}$ & & & & & $\mathrm{X}$ & & & & & \\
\hline & $\begin{array}{c}\text { Eupatorium } \\
\text { squalidum DC. }\end{array}$ & & $\mathrm{X}$ & & & & & & $\mathrm{X}$ & & & $\mathrm{X}$ \\
\hline & Mikania sp. & & $\mathrm{X}$ & & & & & & $\mathrm{X}$ & & & $\mathrm{X}$ \\
\hline Balanophoraceae & $\begin{array}{c}\text { Langsdorffia } \\
\text { hypogaea Mart. }\end{array}$ & & & & & $\mathrm{X}$ & $\mathrm{X}$ & $\mathrm{X}$ & & & & \\
\hline \multirow[t]{8}{*}{ Bignoniaceae } & $\begin{array}{c}\text { Anemopaegma } \\
\text { chamberlaynii } \\
\text { (Sims) Bureau \& } \\
\text { K. Schum }\end{array}$ & & & & $\mathrm{x}$ & & & & & & & $\mathrm{X}$ \\
\hline & $\begin{array}{c}\text { Arrabidaea } \\
\text { brachypoda (DC.) } \\
\text { Bureau }\end{array}$ & & & & $\mathrm{X}$ & & & & $\mathrm{X}$ & & & \\
\hline & $\begin{array}{l}\text { Amphilophium } \\
\text { paniculatum (L.) } \\
\text { Kunth }\end{array}$ & & & & $\mathrm{x}$ & & & & $\mathrm{X}$ & & & $\mathrm{X}$ \\
\hline & $\begin{array}{c}\text { Cybistax } \\
\text { antisyphilitica } \\
\text { (Mart.) Mart. ex } \\
\text { DC. }\end{array}$ & & & $\mathrm{X}$ & & & $\mathrm{X}$ & $\mathrm{X}$ & & & & \\
\hline & $\begin{array}{l}\text { Handroanthus } \\
\text { aureus Mattos }\end{array}$ & & & $\mathrm{X}$ & & & $\mathrm{X}$ & $\mathrm{X}$ & & & & \\
\hline & $\begin{array}{c}\text { Handroanthus } \\
\text { impetiginosus } \\
\text { (Mart. ex DC.) } \\
\text { Mattos }\end{array}$ & & & $\mathrm{x}$ & & & & $\mathrm{x}$ & & & $\mathrm{x}$ & \\
\hline & $\begin{array}{c}\text { Jacaranda } \\
\text { decurrens Cham. }\end{array}$ & & $\mathrm{X}$ & & & & $\mathrm{X}$ & & & & & \\
\hline & $\begin{array}{l}\text { Memora peregrina } \\
\text { (Miers) Sandwith }\end{array}$ & & & & $\mathrm{x}$ & & $\mathrm{X}$ & & $\mathrm{X}$ & & & \\
\hline \multirow[t]{2}{*}{ Blechnaceae } & Blechnum sp. & $\mathrm{X}$ & & & & & & & & $\mathrm{X}$ & & \\
\hline & Salpichlaena sp. & $\mathrm{X}$ & & & & & & & & & & $\mathrm{X}$ \\
\hline \multirow[t]{2}{*}{ Boraginaceae } & $\begin{array}{c}\text { Cordia glabrata } \\
\text { (Mart.) A. DC. }\end{array}$ & & & $\mathrm{X}$ & & & & & & & $\mathrm{X}$ & \\
\hline & Tournefortia sp. & & & & $\mathrm{X}$ & & & & $\mathrm{X}$ & & & $\mathrm{X}$ \\
\hline Burseraceae & $\begin{array}{c}\text { Protium } \\
\text { heptaphyllum } \\
\text { (Aubl.) Marchand }\end{array}$ & & & $\mathrm{X}$ & & & $\mathrm{X}$ & $\mathrm{X}$ & & & & \\
\hline
\end{tabular}




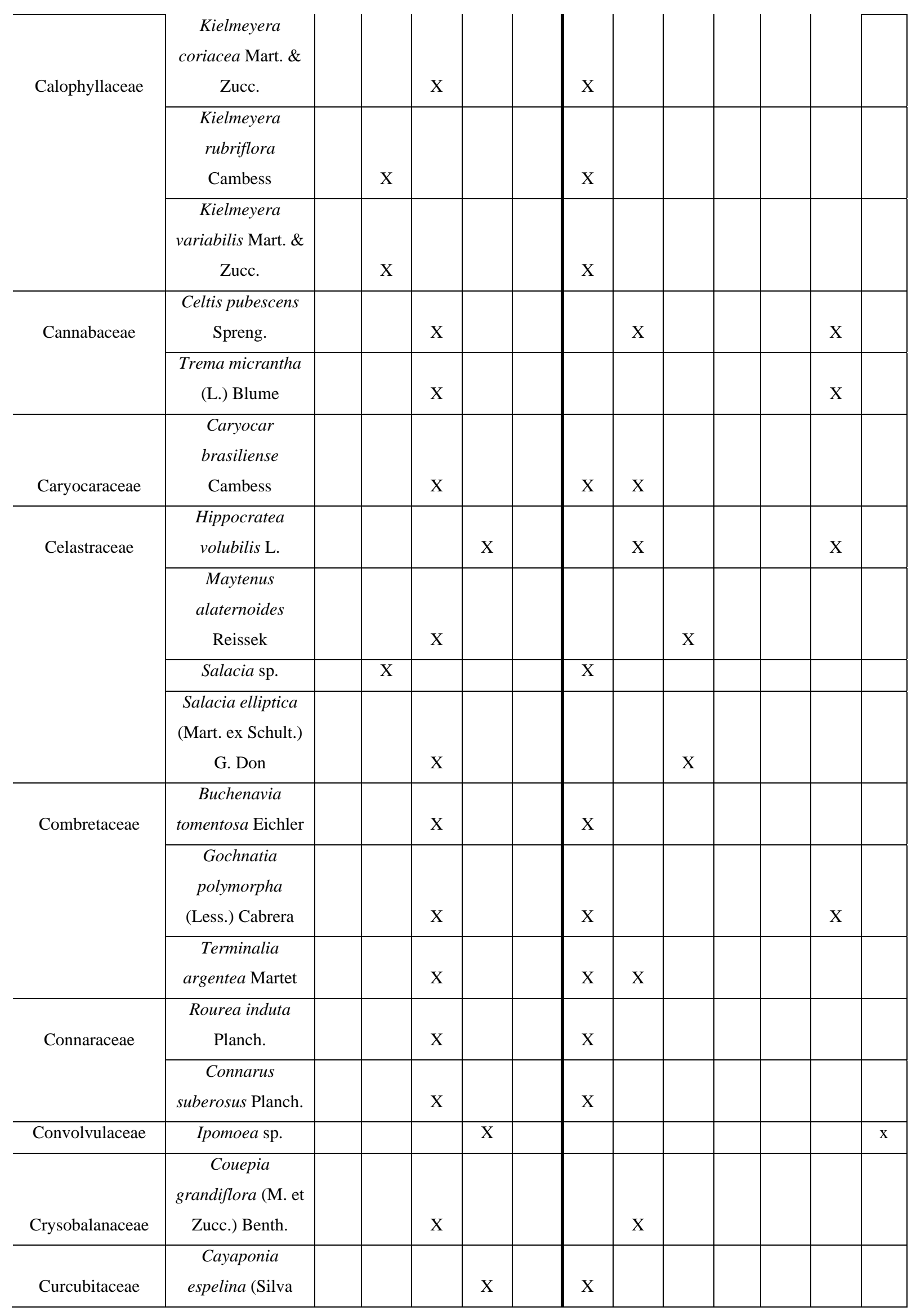




\begin{tabular}{|c|c|c|c|c|c|c|c|c|c|c|c|}
\hline & Manso) Cogn. & & & & & & & & & & \\
\hline \multirow[t]{2}{*}{ Cyperaceae } & Cyperus sp. & $\mathrm{X}$ & & & & & & & $\mathrm{X}$ & & \\
\hline & $\begin{array}{l}\text { Rhynchospora } \\
\text { exaltata Kunth }\end{array}$ & $\mathrm{x}$ & & & & & & & $\mathrm{X}$ & & \\
\hline \multirow[t]{2}{*}{ Dilleniaceae } & $\begin{array}{c}\text { Curatella } \\
\text { americana L. }\end{array}$ & & & $\mathrm{x}$ & & $\mathrm{x}$ & & & & & \\
\hline & $\begin{array}{c}\text { Davilla rugosa } \\
\text { Poir. }\end{array}$ & & & & $\mathrm{X}$ & $\mathrm{x}$ & & & & & \\
\hline Doiscoreaceae & Dioscorea sp. & & & & $\mathrm{X}$ & $\mathrm{X}$ & & & & & $\mathrm{X}$ \\
\hline Ebenaceae & $\begin{array}{c}\text { Diospyros hispida } \\
\text { A. DC. }\end{array}$ & & & $\mathrm{x}$ & & $\mathrm{x}$ & $\mathrm{X}$ & & & & \\
\hline Erythroxilaceae & $\begin{array}{c}\text { Erythroxylum } \\
\text { suberosum A. St.- } \\
\text { Hil. }\end{array}$ & & & $\mathrm{X}$ & & $\mathrm{X}$ & $\mathrm{X}$ & & & & \\
\hline \multirow[t]{7}{*}{ Euphorbiaceae } & Croton sp. & & $\mathrm{X}$ & & & & & & & & $\mathrm{X}$ \\
\hline & $\begin{array}{c}\text { Croton urucurana } \\
\text { Baill. }\end{array}$ & & & $\mathrm{X}$ & & & & $\mathrm{X}$ & & & \\
\hline & $\begin{array}{c}\text { Dalechampia } \\
\text { adscendes (Muell } \\
\text { Arg.) }\end{array}$ & $\mathrm{X}$ & & & & $\mathrm{x}$ & & & & & \\
\hline & $\begin{array}{c}\text { Maprounea } \\
\text { guianensis Aubl. }\end{array}$ & & & $\mathrm{X}$ & & $\mathrm{X}$ & & & & $\mathrm{X}$ & \\
\hline & $\begin{array}{c}\text { Mabea fistulifera } \\
\text { Mart. }\end{array}$ & & & $\mathrm{x}$ & & $\mathrm{X}$ & & & & & \\
\hline & $\begin{array}{c}\text { Pera echinocarpa } \\
\text { Baill. }\end{array}$ & & & $\mathrm{X}$ & & $\mathrm{X}$ & & & & $\mathrm{X}$ & \\
\hline & $\begin{array}{c}\text { Sapium } \\
\text { glandulatum } \\
\text { (Vell.) Pax. }\end{array}$ & & & $\mathrm{X}$ & & & & & $\mathrm{X}$ & & \\
\hline \multirow[t]{5}{*}{ Fabaceae } & $\begin{array}{c}\text { Anadenanthera } \\
\text { colubrina (Vell.) } \\
\text { Brenan }\end{array}$ & & & $\mathrm{X}$ & & $\mathrm{X}$ & & & & & \\
\hline & $\begin{array}{l}\text { Andira humilis } \\
\text { Mart. ex Benth. }\end{array}$ & & $\mathrm{X}$ & & & $\mathrm{X}$ & & & $\mathrm{X}$ & & \\
\hline & $\begin{array}{c}\text { Acacia polyphylla } \\
\text { DC. }\end{array}$ & & & $\mathrm{x}$ & & & $\mathrm{X}$ & & & & \\
\hline & $\begin{array}{c}\text { Anadenanthera } \\
\text { falcata (Benth.) } \\
\text { Speg. }\end{array}$ & & & $\mathrm{X}$ & & & $\mathrm{X}$ & $\mathrm{X}$ & & & \\
\hline & $\begin{array}{c}\text { Bauhinia } \\
\text { holophylla (Bong.) }\end{array}$ & & $\mathrm{x}$ & & & $\mathrm{x}$ & & & $\mathrm{X}$ & & \\
\hline
\end{tabular}




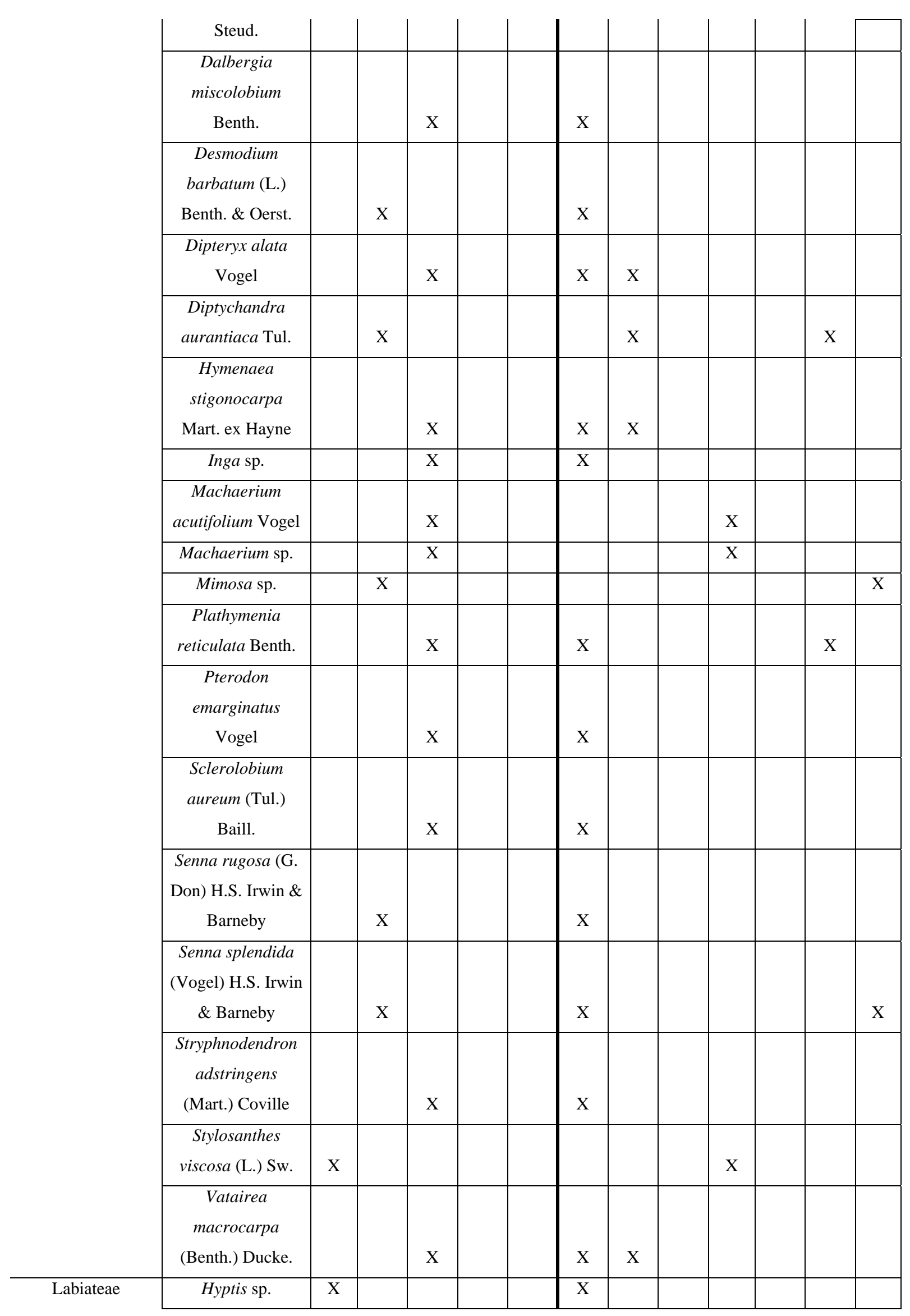




\begin{tabular}{|c|c|c|c|c|c|c|c|c|c|}
\hline & $\begin{array}{l}\text { Hyptis latifolia } \\
\text { Mart.ex Benth. }\end{array}$ & $\mathrm{X}$ & & & $\mathrm{x}$ & & & & \\
\hline Lamiales & Aegyphyla sp. & & $\mathrm{X}$ & & $\mathrm{X}$ & & & & \\
\hline \multirow[t]{5}{*}{ Lauraceae } & $\begin{array}{c}\text { Aiouea trinervis } \\
\text { Meisn. }\end{array}$ & $\mathrm{X}$ & & & $\mathrm{x}$ & & & & \\
\hline & $\begin{array}{c}\text { Endlicheria } \\
\text { paniculata } \\
\text { (Spreng.) J. F. } \\
\text { Macbr. }\end{array}$ & & $\mathrm{X}$ & & & & & $\mathrm{X}$ & \\
\hline & Nectandra sp. & & $\mathrm{X}$ & & & $\mathrm{X}$ & & & \\
\hline & $\begin{array}{c}\text { Ocotea lancifolia } \\
\text { (Schott) Mez }\end{array}$ & & $\mathrm{X}$ & & & $\mathrm{X}$ & & & \\
\hline & $\begin{array}{l}\text { Ocotea densiflora } \\
\text { (Meisn.) Mez }\end{array}$ & & $\mathrm{X}$ & & & & $\mathrm{X}$ & & \\
\hline Lecythidaceae & $\begin{array}{c}\text { Cariniana } \\
\text { estrellensis } \\
\text { (Raddi) Kuntze }\end{array}$ & & $\mathrm{X}$ & & & & $\mathrm{X}$ & & \\
\hline \multirow[t]{2}{*}{ Loganiaceae } & $\begin{array}{c}\text { Strychnos } \\
\text { pseudoquina A. } \\
\text { St.-Hil. }\end{array}$ & & $\mathrm{X}$ & & $\mathrm{x}$ & $\mathrm{X}$ & & & $\mathrm{X}$ \\
\hline & $\begin{array}{c}\text { Strychnos } \\
\text { fulvotomentosa } \\
\text { Gilg. }\end{array}$ & $\mathrm{X}$ & & & $\mathrm{X}$ & & & & \\
\hline Lythraceae & $\begin{array}{l}\text { Lafoensia pacari } \\
\text { A. St.-Hil. }\end{array}$ & & $\mathrm{X}$ & & $\mathrm{x}$ & & & & \\
\hline \multirow[t]{4}{*}{ Malpighyaceae } & $\begin{array}{c}\text { Byrsonima } \\
\text { intermédia A. } \\
\text { Juss. }\end{array}$ & $\mathrm{X}$ & & & $\mathrm{X}$ & & & & \\
\hline & $\begin{array}{c}\text { Byrsonima } \\
\text { verbascifolia (L.) } \\
\text { DC. }\end{array}$ & & $\mathrm{X}$ & & $\mathrm{x}$ & & & & \\
\hline & $\begin{array}{c}\text { Mascagnia } \\
\text { cordifolia (A. } \\
\text { Juss.) Griseb. }\end{array}$ & & & $\mathrm{X}$ & $\mathrm{X}$ & & & & $\mathrm{x}$ \\
\hline & $\begin{array}{c}\text { Peixotoa } \\
\text { cordistipula A. } \\
\text { Juss. }\end{array}$ & & & $\mathrm{X}$ & $\mathrm{X}$ & & & & \\
\hline Malvaceae & $\begin{array}{c}\text { Eriotheca } \\
\text { pubescens (Mart. } \\
\text { \& Zucc.) Schott \& } \\
\text { Endl. }\end{array}$ & & $\mathrm{X}$ & & $\mathrm{X}$ & & & & \\
\hline
\end{tabular}




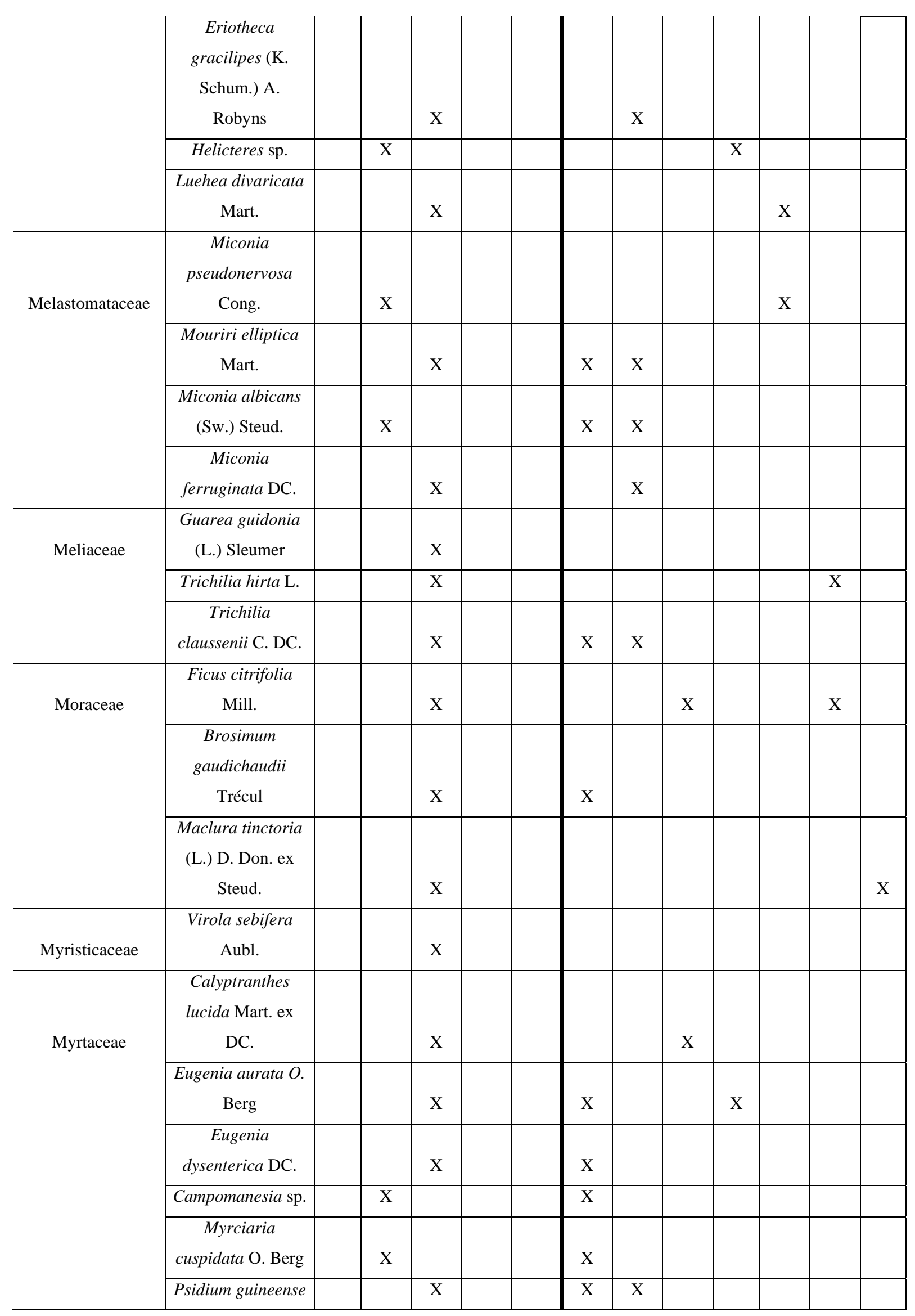

COMPOSIÇÃO FLORÍSTICA DO PARQUE ESTADUAL... 


\begin{tabular}{|c|c|c|c|c|c|c|c|c|c|c|c|}
\hline & Sw. & & & & & & & & & & \\
\hline Nyctaginaceae & $\begin{array}{l}\text { Guapira noxia } \\
\text { (Netto) Lundell }\end{array}$ & & & $\mathrm{x}$ & & & $\mathrm{X}$ & & & & \\
\hline Ochnaceae & $\begin{array}{c}\text { Ouratea } \\
\text { spectabilis (Mart. } \\
\text { ex Engl.) Engl. }\end{array}$ & & & $\mathrm{X}$ & & $\mathrm{X}$ & & & & & \\
\hline Oxalidaceae & $\begin{array}{c}\text { Oxalis physocalyx } \\
\text { Zucc.ex Progel }\end{array}$ & & $\mathrm{X}$ & & & & & & & & $\mathrm{x}$ \\
\hline \multirow[t]{3}{*}{ Passifloraceae } & $\begin{array}{c}\text { Mitostemma } \\
\text { brevifilis Gontsch. }\end{array}$ & & & & $\mathrm{X}$ & & & & & & $\mathrm{x}$ \\
\hline & Passiflora sp. & & & & $\mathrm{X}$ & & & & & & $\mathrm{x}$ \\
\hline & $\begin{array}{c}\text { Passiflora } \\
\text { suberosa L. }\end{array}$ & & & & $\mathrm{X}$ & $\mathrm{x}$ & & & & & $\mathrm{x}$ \\
\hline \multirow[t]{2}{*}{ Piperaceae } & $\begin{array}{c}\text { Piper arboreum } \\
\text { Aubl. }\end{array}$ & & & $\mathrm{X}$ & & & & & & & \\
\hline & Piper aduncum L. & & $\mathrm{X}$ & & & & & $\mathrm{X}$ & & & \\
\hline \multirow[t]{2}{*}{ Primulaceae } & $\begin{array}{c}\text { Rapanea } \\
\text { guianensis Aubl. }\end{array}$ & & & $\mathrm{X}$ & & $\mathrm{x}$ & $\mathrm{X}$ & & & & $\mathrm{x}$ \\
\hline & $\begin{array}{c}\text { Rapanea } \\
\text { ferruginea (Ruiz } \\
\text { \& Pav.) Mez }\end{array}$ & & & $\mathrm{x}$ & & & & & $\mathrm{x}$ & & \\
\hline Proteaceae & $\begin{array}{l}\text { Roupala Montana } \\
\text { Aubl. }\end{array}$ & & & $\mathrm{X}$ & & $\mathrm{x}$ & $\mathrm{X}$ & & & & \\
\hline \multirow[t]{6}{*}{ Rubiaceae } & $\begin{array}{c}\text { Alibertia edulis } \\
\text { (Rich.) A. Rich. ex } \\
\text { DC. }\end{array}$ & & & $\mathrm{X}$ & & $\mathrm{x}$ & $\mathrm{X}$ & & & & \\
\hline & $\begin{array}{c}\text { Alibertia } \\
\text { macrophylla K. } \\
\text { Schum. }\end{array}$ & & & $\mathrm{x}$ & & $\mathrm{x}$ & $\mathrm{X}$ & & & & \\
\hline & $\begin{array}{c}\text { Coussarea } \\
\text { hydrangaefolia } \\
\text { (Benth.) Benth. \& } \\
\text { Hook. f. ex Müll. } \\
\text { Arg. }\end{array}$ & & & $\mathrm{x}$ & & & $\mathrm{X}$ & & & $\mathrm{X}$ & \\
\hline & $\begin{array}{l}\text { Chomelia obtusa } \\
\text { Cham. \& Schltdl. }\end{array}$ & & & $\mathrm{X}$ & & & $\mathrm{X}$ & & & & \\
\hline & $\begin{array}{l}\text { Coccocypselum } \\
\text { lanceolatum (Ruiz } \\
\text { \& Pav.) Pers. }\end{array}$ & $\mathrm{X}$ & & & & & & & $\mathrm{x}$ & & \\
\hline & $\begin{array}{c}\text { Palicourea rígida } \\
\text { Kunth }\end{array}$ & & $\mathrm{X}$ & & & $\mathrm{x}$ & & & & & \\
\hline
\end{tabular}




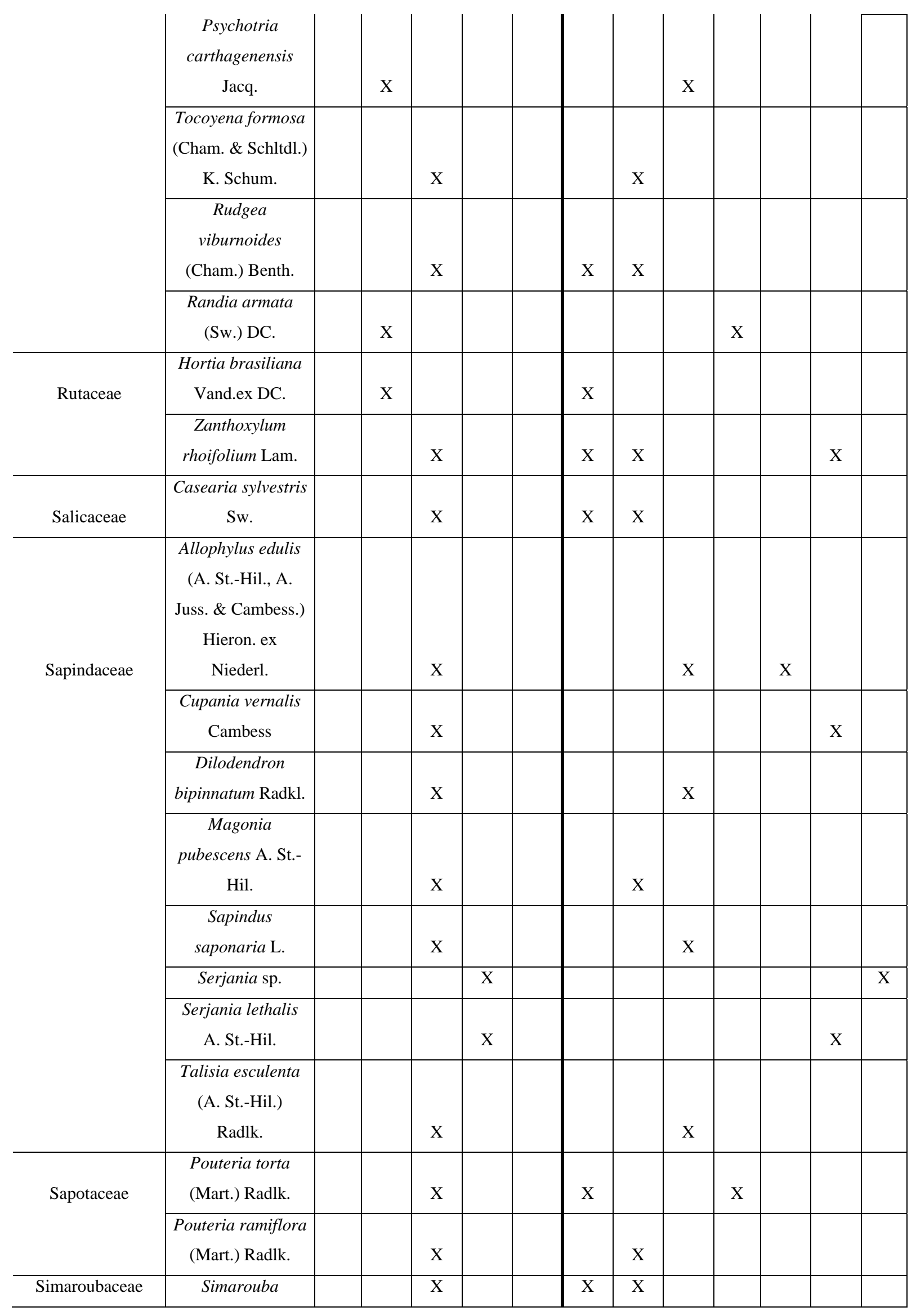

COMPOSIÇÃO FLORÍSTICA DO PARQUE ESTADUAL... 


\begin{tabular}{|c|c|c|c|c|c|c|c|c|c|c|c|}
\hline & $\begin{array}{c}\text { versicolor A. St.- } \\
\text { Hill. }\end{array}$ & & & & & & & & & & \\
\hline Siparunaceae & $\begin{array}{c}\text { Siparuna } \\
\text { guianensis Aubl. }\end{array}$ & $\mathrm{X}$ & & & & $\mathrm{X}$ & & & & & \\
\hline \multirow[t]{2}{*}{ Smilacaceae } & $\begin{array}{c}\text { Smilax campestris } \\
\text { Griseb. }\end{array}$ & & & $\mathrm{X}$ & $\mathrm{X}$ & & & $\mathrm{X}$ & & & \\
\hline & $\begin{array}{l}\text { Smilax fluminensis } \\
\text { Steud. }\end{array}$ & & & $\mathrm{X}$ & $\mathrm{X}$ & & & & & $\mathrm{X}$ & \\
\hline \multirow[t]{3}{*}{ Solanaceae } & $\begin{array}{c}\text { Cestrum } \\
\text { strigilatum R. et P. }\end{array}$ & $\mathrm{X}$ & & & & & $\mathrm{X}$ & & & & $\mathrm{x}$ \\
\hline & $\begin{array}{c}\text { Cestrum } \\
\text { calycinum Kunth }\end{array}$ & $\mathrm{X}$ & & & & & $\mathrm{X}$ & & & & \\
\hline & $\begin{array}{c}\text { Solanum } \\
\text { lycocarpum A. St.- } \\
\text { Hil. }\end{array}$ & $\mathrm{X}$ & & & $\mathrm{X}$ & & & & & & \\
\hline Styracaceae & $\begin{array}{l}\text { Styrax ferrugineus } \\
\text { Nees \& Mart. }\end{array}$ & & $\mathrm{X}$ & & $\mathrm{X}$ & $\mathrm{X}$ & & & & & \\
\hline Urticaceae & $\begin{array}{c}\text { Cecropia } \\
\text { pachystachya } \\
\text { Trécul }\end{array}$ & & $\mathrm{X}$ & & & & & & $\mathrm{X}$ & & $\mathrm{x}$ \\
\hline \multirow[t]{2}{*}{ Verbenaceae } & $\begin{array}{c}\text { Aloysia virgata } \\
\text { (Ruiz \& Pav.) } \\
\text { Juss. }\end{array}$ & & $\mathrm{X}$ & & & & & & & $\mathrm{X}$ & \\
\hline & $\begin{array}{c}\text { Lantana camara } \\
\text { L. }\end{array}$ & $\mathrm{X}$ & & & & $\mathrm{X}$ & & & & & \\
\hline Vitaceae & Cissus erosa Rich. & & & $\mathrm{X}$ & $\mathrm{X}$ & $\mathrm{X}$ & & & & $\mathrm{X}$ & \\
\hline \multirow[t]{3}{*}{ Vochysiaceae } & Callisthene sp. & & $\mathrm{X}$ & & $\mathrm{X}$ & $\mathrm{X}$ & & & & & \\
\hline & $\begin{array}{c}\text { Qualea parviflora } \\
\text { Mart. }\end{array}$ & & $\mathrm{X}$ & & $\mathrm{X}$ & & & & & & \\
\hline & $\begin{array}{c}\text { Vochysia } \\
\text { tucanorum Mart. }\end{array}$ & & $\mathrm{X}$ & & $\mathrm{X}$ & $\mathrm{X}$ & & & & $\mathrm{X}$ & \\
\hline
\end{tabular}

Apenas dois gêneros apresentam três espécies (Kielmeyera e Miconia) e, outros 24 gêneros, duas espécies. A maior parte dos gêneros encontrados (134) possui apenas uma espécie, indicando, em termos de distribuição de gêneros, equidade no local.

O pequeno número de espécies encontradas em ambas as área de Mata de Galeria (16,5\%) e Mata Seca Semidecídua (11,7\%) também são um indicativo do processo de antropização do local, pois estes tipos de formação são, normalmente, mais sensíveis a ação antrópica, que reduz sua diversidade através da retirada seletiva de espécies.

As espécies de maior porte e diâmetro encontradas no local são remanescentes da vegetação original, porém isoladas em alguns pontos da área, como por exemplo, indivíduos de Cariniana estrellensis e Hymenaea stigonocarpa, encontrados em áreas de Cerradão e Mata Seca Semidecídual. Nestes locais também é notada a presença de espécies consideradas secundárias e clímax, indicando que as condições ambientais (fertilidade do Ademir Kleber Morbeck de Oliveira e Ubirazilda Maria Rezende 
solo, umidade relativa, velocidade do vento, etc) já são mais propicias ao desenvolvimento ou manutenção de uma vegetação mais exigente em termos ambientais. Pode-se citar, entre outras, a presença de Dendropanax cuneatus, Eugenia dysenterica, Handroanthus impetiginosus, Myracrodruon urundeuva, Protium heptaphyllum e Trichilia clausseni. Também o processo de recuperação natural da área é bastante intenso, com a presença de banco de plântulas, indicando que o processo de sucessão está ocorrendo.

Porém o replantio de espécies nativas e não nativas, feito anteriormente por policiais militares ambientais, no período em que o local era considerado Jardim Botânico, com o intuíto de recuperar e acelerar o processo de regeneração da área, principalmente de locais atingidos por incêndios ou muito degradados, modificou a estrutura fitossociológica que normalmente seria encontrada neste tipo de vegetação. A presença de espécies condicionadas pela ação antrópica propiciou uma interação de diversas espécies que normalmente não seriam encontradas no mesmo ecossistema, com exceção de alguns ecótonos. Porém com o decorrer dos anos e a proteção do Parque, o processo natural deverá ser dominante e algumas espécies, após completado seu ciclo de vida, desaparecerão ou ficarão restritas a determinados pontos do Parque.

\section{CONCLUSÕES}

O Parque Estadual Matas do Segredo apresenta-se em estágio adiantado de sucessão, sendo identificadas 188 espécies, pertencentes a 63 famílias, sendo 10 espécies no estrato herbáceo, 38, arbustivo, 111, arbóreo, 24, trepadeiras, além de uma espécie de parasita, com os ambientes de cerrado e cerradão possuindo a maior diversidade.

\section{AGRADECIMENTOS}

Os autores agradecem a Universidade AnhangueraUniderp pelo financiamento do projeto GIP (Grupo Interdisciplinar de Pesquisa) e ao Conselho Nacional de
Desenvolvimento Cientifico e Tecnológico (CNPq), pela bolsa concedida (Produtividade em Pesquisa).

\section{REFERÊNCIAS BIBLIOGRÁFICAS}

APG II (Angiosperm Phylogeny Group). An update of the angiosperm phylogeny group classification of the orders and families of flowering plants: APGII. Botanical Journal of the Linnean Society, v. 141, n. 4, p. 399-436, 2003.

CAMPO GRANDE (Município). Campo Grande - 100 anos de construção. Campo Grande: Matriz Editora, 1999. 420p.

HERINGER, E. P.; BARRoso, G. M.; RIZZO, J. A.; RIZZINI, C. T. A flora do cerrado. In: FERRI, M. G. (Coord). IV Simpósio sobre o cerrado. São Paulo: EDUSP; Belo Horizonte: Itatiaia, p. 211-232. 1977.

KÖEPPEN, W. Climatologia: con um estúdio de los climas de la Tierra. Cidade do México: Fondo de Cultura Economica, 1948. 478p.

LORENZI, H. Plantas daninhas do Brasil: terrestres, aquáticas, parasitas, tóxicas e medicinais. 4ed. Nova Odessa: Instituto Plantarum de Estudos da Flora, 2008a. 672p.

LORENZI, H. Árvores brasileiras: manual de identificação e cultivo de plantas arbóreas nativas do Brasil. V. 1. Nova Odessa: Instituto Plantarum de Estudos da Flora, 2008b. 384p. 
LORENZI, H. Árvores brasileiras: manual de identificação e cultivo de plantas arbóreas nativas do Brasil. Vol. 2. Nova Odessa: Instituto Plantarum de Estudos da Flora, 2009. 384p.

MACEDO, S. S.; SAKATA, F. G. Parques urbanos no Brasil. $2^{\text {a }}$ ed. São Paulo: EDUSP/ Imprensa Oficial do Estado de São Paulo, 2003. 206p.

MANTOVAnI, M. (Org.). Parques urbanos e meio ambiente: desafios de uso. 1. ed. São Paulo: Parque Cientec/USP; PEFI, 2006. v. 1. 392p.

MATO GROSSO DO SUL. (Estado). Secretaria de Planejamento e Coordenação Geral. Atlas Multirreferencial. Mapas, 1990. 28p.

MATO GROSSO DO SUL. (Estado). Decreto nº 9.935. Diário Oficial nº 5.279, p. 5, de 6 de junho de 2000.

SANO, S. M.; ALMEIDA, S.P. (Ed.). Cerrado: ambiente e flora. Planaltina: Embrapa-CPAC, 1998. 556p.

SÃO PAULO (Município). Secretaria Municipal do Verde e Meio Ambiente - SVMA. Atlas ambiental do município de São Paulo - O verde, o território, o ser humano: diagnóstico e bases para a definição de políticas públicas para as áreas verdes no município de São Paulo. São Paulo: Fundação Biblioteca Nacional, 2004, 266p.

SCARIOT, A.; SOUSA-SILVA, J. C.; FELFILI, J. M. (Org.). Cerrado: ecologia, biodiversidade e conservação. Brasília: MMA, 2005. 439p.

TOLEDO, F. S.; SANTOS, D. G. Espaço livre de construção - um passeio pelos parques urbanos. Revista da Sociedade Brasileira de Arborização Urbana, v. 7, n. 2, p. 10-23, 2012.

TROPPMAIR, H; GALINA, M. H. Áreas verdes. Revista Território \& Cidadania, n. 2, 2003. 